\title{
Improvement of Biodegradability and Biocompatibility of Electrospun Scaffolds of Poly(Butylene Terephthalate) by Incorporation of Sebacate Units
}

\author{
Nina Heidarzadeh ${ }^{1}$, Luis J. del Valle ${ }^{2,3^{*}}$, Lourdes Franco ${ }^{2,3}$, \\ Jordi Puiggalí ${ }^{2,3 *}$
}

\footnotetext{
${ }^{1}$ Department of Polymer Engineering and Color Technology, Amirkabir University of Technology, PO Box 15875-441, Tehran, IRAN

${ }^{2}$ Chemical Engineering Department, Escola d'Enginyeria de Barcelona Est-EEBE, Av. Eduard Maristany 10-14, Barcelona 08019, SPAIN

${ }^{3}$ Barcelona Research Center for Multiscale Science and Engineering. Universitat Politècnica de Catalunya, Escola d'Enginyeria de Barcelona Est-EEBE, Av. Eduard Maristany 10-14, Barcelona 08019, SPAIN
}

*Corresponding Author:

E-mail: luis.javier.del.valle@upc.edu

E-mail: Jordi.puiggali@upc.edu 


\begin{abstract}
Incorporation of aliphatic units to poly(butylene terephthalate) (PBT) gives rise to biodegradable copolymers with tunable properties (e.g., degradability), depending on the selected comonomer and the specific composition. Specifically, a low molecular weight poly(butylene sebacate-co-terephthalate) (PBSeT) with a high ratio of aliphatic sebacate units (i.e., 70 mol-\% with respect to the total dicarboxylate content) has been employed in this work to get new electrospun biodegradable scaffolds. Appropriate electrospinning conditions have been found despite the limited copolymer molecular weight. In addition, PBSeT has been employed to improve biocompatibility and biodegradability of scaffolds based on the PBT homopolymer. Scaffolds with different properties have been prepared following two strategies: Electrospinning of single solutions of PBT and PBSeT mixtures and co-electrospinning of independent PBT and PBSeT solutions. Characterization involved spectroscopic (FTIR, NMR), calorimetric (DSC, TGA) and surface hydrophobicity analyses. Hydrolytic and enzymatic degradation studies demonstrated the success of the approach due to the susceptibility of the PBSeT component towards the enzymatic attack with lipases from Pseudomonas cepacia and even towards high temperature hydrolysis.
\end{abstract}

Keywords: Poly(butylene terephthalate), poly(butylene sebacate), aliphatic/aromatic copolymers, electrospinning, biodegradability, biocompatibility 


\section{Introduction}

Electrospinning is attracting great attention since last decades because allows manufacturing polymer micro/nanofibers with high surface-to-volume ratio. ${ }^{1-5}$ These fibers are ideal for a broad range of applications, including tissue engineering materials, ${ }^{6}$ sensors, ${ }^{7}$ protective clothing ${ }^{8}$ and filters. ${ }^{9}$ The electrospinning process is characterized by great design variability, being especially relevant to combine different polymers in a single scaffold. In this way, electrospinning can be performed from sol-gel mixtures, ${ }^{10,11}$ homogeneous single solutions ${ }^{12-14}$ and independent solutions to render mixture of fibers ${ }^{15-}$ 18 and coaxial fibers with well differentiated core-shell structures. ${ }^{19-25}$

Biomedical applications of poly(butylene terephthalate) (PBT) and in general of aromatic polyesters are restricted due to their low biodegradation rate. Therefore, it is an attractive challenge to modify the aromatic character of a polymer chain by a random incorporation of aliphatic units with the main goal of getting optimized properties. It is desirable to attain a satisfactory rate of biodegradation while good mechanical properties are retained. ${ }^{26.27}$ Nonwoven fibers of PBT have extensively been used as filtration medium, ${ }^{28}$ composite materials, ${ }^{29}$ and even in some cases as tissue scaffolds ${ }^{30-32}$ due to their low cost and good thermal and mechanical properties. Fibers can be fabricated by different techniques such as melt blowing, ${ }^{33}$ melt $^{34}$ and force spinning, ${ }^{35}$ and electrospinning. ${ }^{36}$

Examples concerning the specific use of nonwoven PBT fibers for tissue engineering scaffolds are abundant in the literature. ${ }^{37-40}$ Electrospun PBT fibers have specifically been proposed as blood vessels for tissue engineering applications ${ }^{37}$ due to its probed biocompatibility and support efficiency for the growth of endothelial cells. ${ }^{41}$ Surface of PBT nonwoven fibers has in some cases been modified in order to improve 
performance. Thus, a simple alkaline and fluorination treatment allows obtaining filters for leukocyte removal. ${ }^{42}$

Electrospinning of degradable PBT copolymers incorporating aliphatic units has not been performed and even works relative to aliphatic poly(alkylene dicarboxylate)s are scarce with the exception of poly(butylene succinate) (Bionolle $\left.{ }^{\circledR}\right) .{ }^{21,43-45}$ Nevertheless, it is clear that aromatic/aliphatic copolymers are highly promising and deserve further studies on their potential applications in the biomedical field. To this end, the present work is centered in the enhancement of biodegradability and biocompatibility of PBT scaffolds by the incorporation of a low molecular weight aliphatic/aromatic copolymer constituted by 1,4-butanediol (B), sebacic acid (Se) and terephthalic acid (T) units (named as PBSeT, see Figure 1). Specifically, three main steps have been considered: a) Preparation of electrospun scaffolds from PBSeT having a high ratio of aliphatic sebacate units (i.e., 70 mol-\% with respect to the total dicarboxylate content), b) Preparation of scaffolds based on single electrospinning of PBT/PBSeT mixtures and c) Preparation of scaffolds by dual electrospinning of PBT and PBSeT. Sebacate units have been selected to be incorporated into the PBT chain since have a similar hydrophobicity than terephthalate units and consequently the change of properties will be mainly a result of the presence of aliphatic ester bonds.

\section{Experimental}

\subsection{Materials}

A PBT sample with number average molecular weight of 12,000 g/mol was purchased from BASF. PBSeT with number and weight average molecular weights of 8,600 and $19,300 \mathrm{~g} / \mathrm{mol}$ were synthesized according to a two step polycondensation process ${ }^{27}$ as described in the supplementary information section. Terephthalic acid (TA) (Shahid 
Tondgooyan Petrochemical Complex, Mahshahr, Iran), sebacic acid (SeA) and 1,4butanediol (BDO) were supplied by Daejung Chemical \& Metal Co., Ltd, Korea. Titanium tetrabutoxide (TBT) as the polycondensation catalyst was purchased from Merck Co., Darmstadt, Germany.

Fibroblast NRK cells from normal rat kidney and epithelial-like MDCK cells from canine cocker spaniel kidney were obtained from ATCC (USA).

\subsection{Electrospinning}

Single electrospinning was used to prepare micro/nanofibers of PBT and PBSeT as well as from their $50 w t-\%$ mixture (named as PBT/PBSeT). A dual electrospinning system (Figure 1) was employed to get scaffolds constituted by a mixture of PBT and PBSeT microfibers. Samples will be named in this case co(PBT-PBSeT).

Electrospun fibers were collected on a plane grounded collector (for single electrospinning) and on a drum rotatory collector operating at $30 \mathrm{rpm}$ (for coelectrospinning). Distances between needle (inside diameter of $0.84 \mathrm{~mm}$ ) and collector were variable between 5 and $15 \mathrm{~cm}$. For co-electrospinning needles were placed equidistantly and facing each other at each side of the drum collector in order to minimize disturbing effects between the two electrically charged jets of PBT and PBSeT solutions. The voltage was varied between 10 and $30 \mathrm{kV}$ and applied to the collector using one/two high-voltage suppliers (Gamma High Voltage Research, ES30-5W). Polymer solutions were delivered via one/two KDS100 infusion syringe pumps (KD Scientific, USA) to control the flow rate (from 0.5 to $10 \mathrm{~mL} / \mathrm{h}$ ). All electrospinning experiments were carried out at room temperature. Electrospun fibers were prepared using an appropriate mixture of solvents and optimized operational parameters (e.g. needle-collector distance, voltage, flow rates and polymer concentrations). 


\subsection{Measurements}

Infrared absorption spectroscopic data were acquired with a FTIR 4100 Jasco spectrometer (Tokyo, Japan) dotted with attenuated total reflection (ATR) (Specac model MKII Golden Gate). A heated Diamond Top-Plate was employed to study thermal induced transition.

${ }^{1} \mathrm{H}-\mathrm{NMR}$ spectra were acquired with a Bruker AMX-300 spectrometer (Bremen, Germany) operating at $300.1 \mathrm{MHz}$. Chemical shifts were calibrated using tetramethylsilane as an internal standard. A mixture of deuterated chloroform and trifluoroacetic acid (TFA) $(1: 1 \mathrm{v} / \mathrm{v})$ was used as solvent.

\subsection{Morphology and properties of electrospun scaffolds}

Preliminary observations to evaluate the influence of electrospinning parameters on the fiber morphology were carried out by optical microscopy using a Zeiss Axioskop 40 microscope (Göttingen, Germany). Micrographs were taken with a Zeiss AxiosCam MRC5 digital camera (Göttingen, Germany).

Detailed inspection of texture and morphology of electrospun samples was conducted by scanning electron microscopy using a Focus Ion Beam Zeiss Neon 40 instrument (Carl Zeiss, Germany). Carbon coating was accomplished by using a Mitec K950 Sputter Coater (Quorum Technologies Ltd., Ashford, UK) fitted with a film thickness monitor k150x. Samples were visualized at an accelerating voltage of $5 \mathrm{kV}$. Diameter of electrospun fibers was measured with the SmartTiff software from Carl Zeiss SMT Ltd.

Contact angles (CA) were measured at room temperature with sessile drop method using an OCA-15 plus contact angle microscope (Dataphysics, USA) and SCA20 software. Contact angle values of the right and left sides of distilled water drops were 
measured and averaged. Measurements were performed $10 \mathrm{~s}$ after the drop (5 $\mu \mathrm{L}$ ) was deposited on the sample surface. All CA data were an average of six measurements on different surface locations.

Calorimetric data were obtained by differential scanning calorimetry (DSC) with a TA Instruments Q100 series (New Castle, DE, USA) equipped with a refrigeration cooling system (RCS). Experiments were conducted under a flow of dry nitrogen with a sample weight of approximately $5 \mathrm{mg}$ and calibration was performed with indium. Heating runs were carried out at a rate of $20^{\circ} \mathrm{C} / \mathrm{min}$.

Thermal degradation was studied at a heating rate of $20^{\circ} \mathrm{C} / \mathrm{min}$ with around $5 \mathrm{mg}$ samples in a Q50 thermogravimetric analyzer (TGA) of TA Instruments (New Castle, DE, USA) and under a flow of dry nitrogen. Test temperatures ranged from 50 to $600{ }^{\circ} \mathrm{C}$.

\subsection{Degradation studies}

Degradation studies were performed with the electrospun polymer matrices. In-vitro hydrolytic degradation assays were carried out under accelerated conditions provided by an increase of $\mathrm{pH}$ or temperature from close to physiological conditions (i.e. $\mathrm{pH} 10$ and $37^{\circ} \mathrm{C}$, pH 7 and $70{ }^{\circ} \mathrm{C}$ ). An universal buffer (citrate-phosphate-borate) ${ }^{26,27}$ was prepared by mixing $100 \mathrm{~mL}$ of $0.33 \mathrm{M}$ citric acid, $100 \mathrm{~mL}$ of $0.33 \mathrm{M}$ phosphoric acid, $3.54 \mathrm{~g}$ of boric acid, $343 \mathrm{~mL}$ of $1 \mathrm{M} \mathrm{NaOH}$ and distilled water up to a total volume of $1 \mathrm{~L}$. Buffers at pHs of 7 and 10 were then obtained by mixing $20 \mathrm{~mL}$ of this universal buffer with 32.9 and $18.1 \mathrm{~mL}$ of $0.1 \mathrm{M} \mathrm{HCl}$ and adding distilled water up to a total volume of $100 \mathrm{~mL}$.

Square pieces of $2 \mathrm{~cm} \times 2 \mathrm{~cm}$ and thickness close to $100 \mu \mathrm{m}$ were kept under orbital shaking in bottles filled with $20 \mathrm{~mL}$ of the degradation medium and sodium azide $(0.03 \mathrm{w} / \mathrm{v}-\%)$ to prevent microbial growth for the selected exposure times. The samples were then thoroughly rinsed with distilled water, dried to constant weight under vacuum 
and stored over $\mathrm{P}_{4} \mathrm{O}_{10}$ before analysis. Degradation studies were performed in triplicate and the data were reported according to the average values.

The enzymatic studies were carried out with lipase from Pseudomonas cepacia $(\geq$ $40 \mathrm{U} / \mathrm{mg}$ ) using three replicates. Samples with the above indicated geometry were exposed to $2 \mathrm{~mL}$ of phosphate buffer $\left(19.268 \mathrm{~g}\right.$ of $\mathrm{Na}_{2} \mathrm{HPO}_{4} .12 \mathrm{H}_{2} \mathrm{O}$ and $1.796 \mathrm{~g}$ of $\mathrm{KH}_{2} \mathrm{PO}_{4}$ in $1 \mathrm{~L}$ of deionized water, $\left.\mathrm{pH} 7.4\right)$ containing the enzyme (100 mg/L) along with sodium azide (0.03 w/v-\%) and calcium chloride (5 mM). Solutions were renewed every week to prevent enzymatic activity loss. Samples were extracted, washed and dried as previously described. Scanning electron microscopy (SEM) was employed to examine the morphology of films after different times of exposure to the selected degradation media.

Weight retention $\left(W_{r}\right)$ of the specimens was determined from the weight of samples after $\left(W_{d}\right)$ and before degradation $\left(W_{0}\right)$ (equation 1$)$ :

$$
W_{r}=100 \times W_{d} / W_{0}
$$

\subsection{Cellular adhesion and proliferation assays}

NRK and MDCK cells were cultured in Dulbecco's modified Eagle medium (DMEM) as previously reported. ${ }^{14,15,21,45}$ Pieces of the electrospun scaffolds $(1 \mathrm{~cm} \times 1 \mathrm{~cm} \times 0.1 \mathrm{~mm})$ were placed into each well of a 24-well culture plate. Samples were fixed in the wells with a small drop of silicone (Silbione ${ }^{\circledR}$ MED ADH 4300 RTV, Bluestar Silicones France SAS, Lyon, France) and then sterilized by UV-irradiation in a laminar flux cabinet for 15 min. For the cell adhesion assay, aliquots of $50-100 \mu \mathrm{L}$ containing $5 \times 10^{4}$ cells were seeded onto the electrospun samples in each well and incubated with $1 \mathrm{~mL}$ of the culture medium for $24 \mathrm{~h}$. Cell proliferation assay was performed by a similar procedure, but the 
aliquot of 50-100 mL contained $2 \times 10^{4}$ cells and the cultures were maintained for 4 days to allow cell growth.

Cell viability of samples was evaluated by the MTT assay using three replicates.

The results were averaged. ${ }^{26.27}$ Samples with adhered and grown cells on the scaffolds were fixed with $2.5 \mathrm{w} / \mathrm{v}$-\% formaldehyde at $4{ }^{\circ} \mathrm{C}$ overnight. They were subsequently dehydrated and processed for observation by scanning electron microscopy. In addition, confocal laser scanning microscopy (Carl ZEISS LSM 800) was performed to obtain fluorescence images of the cells grown onto the matrices. To this end, a multicolor staining of the fixed and dehydrated samples was performed as above indicated. The actin filaments were stained with the fluorochrome Atto 488 Phalloidin and then the nuclei were stained with the Hoechst 33258 solution.

\subsection{Statistical analysis}

Experimental values were averaged and graphically represented together with their respective standard deviations. One-way ANOVA test was performed to compare the means of all groups and Tukey's test was applied to determine a statistically significant difference between two groups (Origin Pro V10 software). The differences between the paired percentages were compared using Fisher's exact test (Minitab 18 software). The significant differences were established with a $p$-value $<0.05$.

\section{Results and discussion}

\subsection{Electrospinning of PBT, PBSeT, PBT/PBSeT and co(PBT-PBSeT)}

PBSeT was the most difficult polymer to be electrospun due to its lower molecular weight (i.e. $M_{n}$ values of 8,600 and $12,000 \mathrm{~g} / \mathrm{mol}$ were determined for PBSeT and PBT, respectively). It is well known that stabilization of the spinning jet is difficult when molecular weight is low since the number of entanglements between chains decreases as 
well as the cohesion of the fiber. Therefore, fiber break-up and droplet formation are usual. These problems can be avoided by increasing the polymer concentration in the solution, being the selection of an appropriate solvent a crucial point. In fact, Shenoy et al. indicated that the number of entanglements per chain should be higher than 2.5 to get stable fiber formation. ${ }^{47}$

Optimization of electrospinning parameters was performed following different steps. First, by choosing a common solvent for all the samples, which was mainly conditioned by the PBSeT component as indicated before. Secondly, by selecting the minimum polymer concentration that allowed getting continuous and regular fibers. The third step was the selection of flow rate and applied voltage, which were optimized by successive iterations. The distance between the needle and the collector had a small effect being possible to use a wide distance range when the other parameters were optimized. Table 1 summarizes the chosen conditions for the different samples. A chloroform/acetone $(2: 1 \mathrm{v} / \mathrm{v})$ mixture was found as an appropriate solvent for all samples. As above discussed the required polymer concentration was very high for PBSeT (60 w/v\%), being also required a slight decrease of the flow rate (i.e. $3 \mathrm{~mL} / \mathrm{h}$ with respect to 4 $\mathrm{mL} / \mathrm{h}$ required for PBT) since the increase of this magnitude tends to render higher diameters and increase the risk of fiber breakage. It is interesting to note that the PBT/PBSeT mixture could be electrospun at the same total polymer (i.e. PBT+PBSeT) concentration employed for PBT (i.e. $20 w / v$-\%). This feature means that the presence of the higher molecular weight polymer was enough to make feasible the electrospinning of PBSeT. It should also be pointed out that relatively favorable interactions between PBT and the copolymer could be established due to the common presence of terephthalate units. Parameters determined for the PBT/PBSeT blend were logically intermediate between those found for PBT and PBSeT since assays were carried out at low PBSeT 
concentration. The parameters optimized for each polymer were selected, with the exception of distance, to perform the co-electrospinning process using the drum rotatory collector (Figure 1). Note that in this case, the resulting scaffold should have a higher PBSeT content as consequence of the required high polymer concentration despite the indicated decrease of the flow rate. In fact, the scaffold should have around $69 w t-\%$ of PBSeT in contrast with the lack of limitations for preparing PBT/PBSeT blends. Electrospinning of the polymer mixture appears as the best option when a high PBT content is preferred.

Figures 2a-2d shows the SEM images of PBT, PBSeT, PBT/PBSeT and co(PBTPBSeT) electrospun scaffolds. Micrographs revealed that PBT fibers were continuous, uniform and had a smooth surface (Figure 2a), whereas PBSeT fibers showed incipient bead formation (Figure 2b). These irregular protuberances reflect the greater difficulty to generate continuous fibers with the low molecular weight sample.

Diameter sizes of electrospun PBT and PBSeT fibers were also clearly different (Figure 3) as presumable from the high variation on polymer concentration (i.e. from 20 $w / v$-\% to $60 w / v$-\%) since the change on the flow rate was not significant (i.e. from 4 $\mathrm{mL} / \mathrm{h}$ to $3 \mathrm{~mL} / \mathrm{h}$ ) to lead a compensatory effect. Thus, diameters increased from $0.84 \pm$ $0.42 \mu \mathrm{m}$ to $4.38 \pm 1.97 \mu \mathrm{m}$ when PBT was changed by the lower molecular weight PBSeT copolymer. Relatively uniform and continuous fibers were attained by electrospinning the PBT/PBSeT mixture. In this case, it was necessary to decrease the flow rate up to 1.5 $\mathrm{mL} / \mathrm{h}$ to avoid bead formation when a low polymer concentration was kept (20 w/v-\%). The diameter distribution was narrow and in the nanoscale range (i.e. $0.46 \pm 0.21 \mu \mathrm{m}$ ) as a consequence of the low flow rate. SEM micrographs of the co-electrospun scaffolds clearly revealed two populations of fibers and a bimodal diameter distribution. The corresponding diameter sizes were $0.88 \pm 0.41 \mu \mathrm{m}$ and $4.39 \pm 1.25 \mu \mathrm{m}$ and consequently 
in full agreement with those previously determined from the electrospinning of the single components.

\subsection{Characterization of electrospun PBT, PBSeT, PBT/PBSeT and co(PBT- PBSeT) electrospun scaffolds}

FTIR spectra of prepared scaffolds showed well defined and intense ester bands that could be associated to $\mathrm{C}=\mathrm{O}$ stretching $\left(1717 \mathrm{~cm}^{-1}\right)$, asymmetric and symmetric $\mathrm{C}-\mathrm{O}$ stretching of terephthalate units (1268 and $1100 \mathrm{~cm}^{-1}$, respectively) and asymmetric and symmetric C-O stretching of sebacate units (1219 and $1173 \mathrm{~cm}^{-1}$, respectively). C-O stretching bands were specially interesting since were sensitive to the aromatic and aliphatic content and allowed to differentiate the PBSeT and co(PBT-PBSeT) scaffolds due to the increased content of aromatic groups in the second sample (Figure S1). Relative intensities of peaks in FTIR spectra taken at different zones of the co-electrospun scaffolds were always similar indicating homogeneous compositions. This feature was significant to demonstrate that a uniform fiber distribution was achieved.

Composition of PBT/PBSeT and co(PBT-PBSeT) scaffolds was more exactly analyzed by ${ }^{1} \mathrm{H}-\mathrm{NMR}$ spectroscopy. Figure 4a shows the NMR spectrum of the different scaffolds. The single and well differentiated proton signals of the aromatic and the aliphatic $\mathrm{CH}_{2} \mathrm{C}=\mathrm{O}$ groups at 8.14 and $2.42 \mathrm{ppm}$, respectively, were employed to determine the terephthalate molar fraction $\left(f_{t}\right)$ in the copolymer (equation 2) and the PBT weight percentage in the PBT/PBSeT and co(PBT-PBSeT) electrospun samples (equation 3).

$$
\begin{gathered}
f_{t}=\left(A_{8.14} / 4\right) /\left[\left(A_{8.14} / 4\right)+\left(A_{2.42} / 4\right)\right] \\
\operatorname{PBT}(w t-\%)=100 \times 220 \times\left(\left(A_{8.14} / 4\right)-\left(A_{2.42} / 4 \times f_{t} /\left(1-f_{t}\right)\right)\right) /\left[\left(220 \times\left(\left(A_{8.14} / 4\right)-\right.\right.\right. \\
\left.\left.\left(A_{2.42} / 4 \times f_{t} /\left(1-f_{t}\right)\right)\right)+256 \times\left(A_{2.42} /\left(4 \times\left(1-f_{t}\right)\right)\right)\right]
\end{gathered}
$$


where 220 and 256 are the molecular weights of PBT and PBSeT repeat units, respectively, and $A_{i}$ is the area of the involved NMR signal.

PBT contents of $51 \pm 1 w t-\%$ and $68 \pm 5 w t-\%$ were determined for PBT/PBSeT and co(PBT-PBSeT) scaffolds, respectively. These values were in full agreement with the theoretical values (50wt-\% and $69 w t-\%$ ) deduced from the polymer concentrations in the corresponding electrospun solutions and the applied flow rates. Compositions were evaluated considering four independent samples. Additional sampling of the coelectrospun scaffolds was performed considering four $1 \mathrm{~cm}$ equidistant points along their diagonal.

The $\mathrm{OCH}_{2}$ butanediol (B) protons were highly interesting (Figure 4b) due to their sensitivity to the neighboring dicarboxylic acid units. Thus, the signal was split giving rise to four triplets associated to T-B-T and Se-B-Se homosequences and Se-B-T and TB-Se heterosequences. Intensity of the observed peaks was indicative of a random distribution of terephthalate and sebacate units in the synthesized copolymer. ${ }^{26,27}$ Figure 4b clearly shows the higher ratio of Se-B-Se sequences in the sample prepared by coelectrospinning with respect to that found in the PBT/PBSeT scaffold. This fact is obviously a consequence of the indicated limitations for electrospinning the PBSeT low molecular weight component (i.e., use of a high PBSeT concentration).

\subsection{Thermal properties of electrospun PBT, PBSeT, PBT/PBSeT and co(PBT- PBSeT) electrospun scaffolds}

Intrinsic thermal properties of the different samples can be compared considering the heating scan of melt crystallized samples (Figure 5) where differences derived from processing under distinct conditions (e.g. polymer concentration, flow rate, etc) are avoided. Data summarized in Table 2 shows a semicrystalline behavior with melting peaks associated to the two immiscible phases having aromatic terephthalate units and 
aliphatic sebacate units. ${ }^{28}$ Melting of terephthalate rich phases is characterized by two peaks, which are indicative of different lamellar populations and a reordering process. Note that the copolymer was able to crystallize during the previous cooling run despite having a random distribution and high comonomer content. The melting temperature associated to the aliphatic phase was low and around $45-50{ }^{\circ} \mathrm{C}$, which can be considered a positive factor for enhancing degradation due to the expected mobility of sebacate segments in the amorphous phase ( $T_{g}$ close to $-61.5^{\circ} \mathrm{C}$, not shown). Melting behavior of PBT/PBSeT and co(PBT-PBSeT) was fully consistent with the presence of the two components and their specific content. Thus, enthalpy of the lower temperature peak was higher for the co-electrospun sample with respect to PBT/PBSeT due to its higher content on PBSeT. Cold crystallization peaks were not detected during the heating scans demonstrating that samples were able to crystallize during the cooling from the melt state.

The first heating scan of the electrospun samples (Figure 5) revealed than in all cases both aliphatic and aromatic phases were able to crystallize during processing. This is a logical feature taking into account the capacity of electrospinning to orient polymer chains and favour the crystallization process. Some distinctive points with respect to melt crystallized samples can be mentioned: a) The enthalpy associated to the fusion of the aromatic phase was lower for samples prepared by electrospinning; b) By contrast, The enthalpy corresponding to the aliphatic phase increased for the electrospun samples (note for example the enthalpies of $26 \mathrm{~J} / \mathrm{g}$ and $17 \mathrm{~J} / \mathrm{g}$ determined for the first peak of PBT/PBSeT in the first and second heating runs, respectively). It is clear that sebacate units crystallized with higher difficulty from the melt state since crystalline domains rich on the aromatic component were first crystallized during the cooling run, while both phases could crystallize at the same time during the solvent evaporation step of electrospinning; c) A single melting peak was characteristic for the aromatic phase 
suggesting both a crystallization from solution that gives rise to the thicker lamellae or a very scarce stability of the thinner lamellae (i.e. a complete reordering process giving thicker lamellae took place during heating). This single peak contrasts with the double peak detected for the melt crystallized sample. d) A relative complex fusion of the aliphatic phase since shoulders can be detected in the corresponding melting peak. e) A peculiar heating trace for the co(PBT-PBSeT) sample where a small cold crystallization peak could be detected together with a broad shoulder of the aromatic melting peak that starts around $130{ }^{\circ} \mathrm{C}$. These features indicated that the previous fusion of the aliphatic phase of the PBSeT surrounding fibers affects the capacity of crystallization and fusion of PBT rich segments.

TGA and DTGA traces (Figure S2) showed that all studied samples thermally degraded at a temperature clearly higher than the corresponding melting points. Although decomposition seems to have a highly predominant step (i.e. a single DTGA peak was observed for PBT at $399.8^{\circ} \mathrm{C}$ ), different mechanisms are probably involved, as reported for PBT. ${ }^{48-50}$ In this case, degradation started with an ionic decomposition that resulted in the production of tetrahydrofuran. The subsequent process was associated with ester pyrolysis reactions that yielded 1,3-butadiene at the beginning and finally produced aromatic species. ${ }^{48-50}$ Thermal decomposition for PBSeT proceeded according to two steps (Figure S2b). The first one was the predominant process (i.e. the weight loss was 92-97\%) and occurred at a temperature of $407.6{ }^{\circ} \mathrm{C}$ that was slightly higher than that observed for PBT. ${ }^{48-50}$ The main DTGA peak of PBT/PBSeT and co(PBT-PBSeT) samples appeared at intermediate temperature values (403.2 and $403.9^{\circ} \mathrm{C}$ ) as presumable taking into account their intermediate compositions. Differences in the degradation process of samples having the two polymer components were not significant (Table 3). Therefore, it could be discarded an acceleration of degradation due to a closer contact 
between the two polymer chains or from a smaller diameter of fibers that could facilitate the release of degradation products. Note that both considerations together with a slightly higher content of PBT should favour the degradation of PBT/PBSeT, a feature that was not observed. In any case, the presence of the PBT homopolymer lead to a complete degradation of the copolymer at a slightly lower temperature (i.e. $403.9{ }^{\circ} \mathrm{C}$ with respect to $407.6{ }^{\circ} \mathrm{C}$ ) and even to a suppression of the second degradation step.

Finally, it could be indicated that practically no differences were found between the char yields, although the greatest value was determined for PBT and co(PBT-PBSeT) (i.e. 5.2 and 6.5\%, respectively, which contrast with values of 2.4 and 3.9\% determined for PBSeT and PBT/PBSeT, respectively). This char yield is a direct consequence of the aromatic content of all samples associated to the terephthalate units.

\subsection{Hydrophobicity of electrospun PBT, PBSeT, PBT/PBSeT and co(PBT- PBSeT) electrospun scaffolds}

Characteristics of the scaffold surfaces may have some influences on the attachment and growth of cells and even on the degradation processes. Figure 6 shows that all samples had an hydrophobic character since contact angles were always higher than $90^{\circ}$. Nevertheless, a significant variation was observed depending on the composition, being PBT the most hydrophobic component. Specifically a significant difference of $30^{\circ}(p<$ 0.05) was detected between PBT and PBSeT single component scaffolds. An intermediate hydrophobic character was found for PBT/PBSeT and co(PBT-PBSeT) scaffolds, being the contact angle mainly dependent on the final composition. Thus, angles of $119^{\circ}$ and $100^{\circ}$ were measured for PBT/PBSeT and co(PBT-PBSeT), respectively, being the lower value logically found with the sample with a higher sebacate content (60 wt-\%, see Table $1)$. 
Although fiber morphology could influence the final hydrophobicity due to changes on texture and roughness of the scaffold surface, the effect was not significant. Note that the diameter size was lower for the PBT/PBSeT sample (i.e. a less hydrophobic smooth surface should be expected), while its hydrophobicity was $19^{\circ}$ higher than deduced for the co-electrospun sample. These results clearly demonstrate that the changes in hydrophobicity were due to the presence of PBSeT fibers in the matrices. The obtained results pointed out that the surface of PBSeT and co(PBT-PBSeT) samples should be more favorable for cell colonization as has been reported for other systems in base of hydrophobic/hydrophilic surface properties. ${ }^{21}$

\subsection{Hydrolytic and enzymatic degradability of PBT, PBSeT, PBT/PBSeT and co(PBT-PBSeT) electrospun scaffolds}

All hydrolytical degradation experiments were performed under accelerated conditions defined by a basic $\mathrm{pH}$ of 10 or a high temperature of $70{ }^{\circ} \mathrm{C}$ since it is well known that the PBT major component is practically undegradable under physiological conditions (i.e. $\mathrm{pH} 7.4$ and $37^{\circ} \mathrm{C}$ ). Figures $7 \mathrm{a}$ and $7 \mathrm{~b}$ showed that the effect of $\mathrm{pH}$ was moderate since only PBSeT showed significant evidences of degradation (i.e. weight loss of $15 \%$ after 30 days of exposure, while values lower than $2 \%$ were found for the other samples, and these values were significantly different, $p<0.05$ ). The increase of temperature up to 70 ${ }^{\circ} \mathrm{C}$ had a greater impact on degradation since a regular increase of the weight loss during exposure could be detected for all samples containing PBSeT. Specifically, values of 27\%, 18\% and 9\% were determined after 30 days for PBSeT, co(PBT-PBSeT) and PBT/PBSeT, respectively. Note that this weight loss can be well correlated with the PBSeT content (i.e. the content of sebacate units) in the electrospun scaffold. These results showed significant differences $(p<0.05)$ in the accelerated temperature deposition of the PBSeT and co(PBT-PBSeT) matrices with respect to the PBT matrix; while the 
differences between the matrices of PBT/PBSeT and PBT were borderline $(p=0.06)$. Finally, among the matrices that incorporate sebacate units, the PBSeT matrix was significantly different $(p<0.05)$ with respect to the PBT/PBSeT blend matrix, but not with respect to the co(PBT-PBSeT) matrix where the PBSeT remain pure.

Incorporation of enzymes (e.g., lipase from Pseudomonas cepacia) in the degradation medium gave on the contrary rise to an appreciable degradation, even at 37 ${ }^{\circ} \mathrm{C}$, of samples having the PBSeT component. The weight loss curves followed a similar trend with the maximum and minimum losses detected for PBSeT (15\%) and PBT/PBSeT (7\%), respectively. Note that in all experiments PBT was not degraded (i.e. the weight loss remained lower than $1 \%$ during exposure the enzymatic medium), being demonstrated the efficacy of incorporating the PBSeT copolymer even in a blended form. Finally, the results of the enzymatic degradation occurred in a similar way to the accelerated degradation at $70{ }^{\circ} \mathrm{C}$. Significant differences were demonstrated $(p<0.05)$ in the enzymatic degradation of PBSeT and co(PBT-PBSeT) matrices in comparison to the PBT matrix.

Morphological changes after 30 days of exposure can be observed through the SEM micrographs displayed in Figure 7. Fiber textures were unmodified for PBT scaffolds and even for the degradable PBSeT in the hydrolytic medium at pH 10 (Figures 7d and 7e, respectively). Pore distribution and even the fiber morphology could still clearly be detected for the PBSeT scaffold exposed to hydrolytic medium at $70{ }^{\circ} \mathrm{C}$ (Figure 7f) despite a higher weight loss was achieved (27\%). The micrograph shows also a partial melting of fibers due to the fact that the medium temperature was scarcely higher than the polymer melting temperature. This feature is also observed for the scaffolds prepared by simple and dual electrospinning (Figures $7 \mathrm{~g}$ and $7 \mathrm{~h}$ ). Morphological changes are more visible for samples exposed to the enzymatic degradation medium (Figures 7i-7k) since 
melting effects that can mask texture modifications are avoided. Note that high surface deterioration and even fiber breakage can be observed for scaffolds containing PBT and PBSeT (Figures 7i-7k). Enzymatic attack can be visualized through the formation of surface pores, which are more evident for PBT/PBSeT fibers (Figure 7j), where polymers with different susceptibility to degradation (i.e. PBT and PBSeT) are combined.

Hydrolytic and enzymatic degradation of samples containing PBSeT are consequence of the disruption of its aliphatic ester bonds. Logically, surface and bulk erosion mechanisms should be expected for enzymatic and hydrolytic degradations, respectively. However, the high surface/volume ratio of the electrospun fibers made difficult the distinction between these mechanisms by means of a simple morphologic analysis.

\subsection{Biocompatibility of PBT, PBSeT, PBT/PBSeT and co(PBT-PBSeT) electrospun scaffolds}

Figure 8 shows the adhesion and proliferation results obtained with the different studied samples and using epithelial (MDCK) and fibroblasts (NRK) cell lines. A significant reduction in the viability of both cell lines was observed for the PBT scaffold (Figures 8a and 8b), while a clearly enhanced viability was detected for scaffolds having the PBSeT component. Probably, the increase on cell adhesion and proliferation observed for the PBSeT scaffold is a consequence of its higher hydrophilicity and the increase of the surface roughness caused by the increase of the diameter size. The biocompatible characteristics of the PBT scaffold can therefore be clearly improved by incorporating the new aliphatic/aromatic copolymer by both single and dual electrospinning techniques. Specifically, PBT/PBSeT and co(PBT-PBSeT) scaffolds even had a greater cell viability than the scaffold constituted by only PBSeT. This particular behaviour can be caused by the higher presence of terminal groups in PBSeT due to its lower molecular weight. 
Differences were not significant between PBT/PBSeT and co(PBT-PBSeT) due to two counteracting effects: the higher PBSeT content (i.e., higher ratio of terminal gropus) and the higher hydrophilicity of the co-electrospun scaffold. NRK cell were always more sensitive to cytotoxic effects and led to slightly lower cell adhesion and proliferation.

Figure 9 shows SEM images where the morphology of the epithelial and fibroblast cells cultured in the different electrospun scaffolds can be observed. These qualitative results showed that both cell lines can colonize the new scaffolds as demonstrated by the clear formation of cellular monolayers during proliferation (see Figure 9, and additionally the confocal microscopy images shown in Figure S3). Furthermore, the colonization of these materials was demonstrated by the adhesion of cells on the fibers. Insets of Figure 9 reveal that cells extend their cytoplasm on the fibers and sustain the above indicated data on viability (Figure 8).

\section{Conclusions}

Scaffolds constituted by PBSeT microfibers with a high sebacate content (70 mol-\%) could be obtained by means of the electrospinning technique and using a chloroform/acetone solvent mixture. The main experimental limitation concerned to the high copolymer concentration that was necessary to produce enough chain entanglements to compensate its low molecular weight. Samples were susceptible to both hydrolytic and enzymatic degradation and showed good biocompatibility.

Electrospinning of mixtures of the indicated copolymer and PBT rendered also biocompatible scaffolds susceptible to the enzymatic attack with lipases from Pseudomonas cepacia. Incorporation of PBT allowed working with a low polymer concentration and get fibers in a nanometric scale that could enhance degradability. Electrospun fibers were characterized by phase separation and the presence of significant 
crystalline domains of PBSe and PBT. Results demonstrated that the incorporation of the aliphatic/aromatic copolymer was effective to change properties of PBT scaffolds and specifically to increase their hydrophilicity, biocompatibility and biodegradability.

Coelectrospinning was an effective technique to get biodegradable and biocompatible scaffolds constituted by PBSeT and PBT where the fiber characteristics of each component could be mantained.

\section{Acknowledgements.}

J.P., L.J.D.V. and L.F. are thankful for the supports from MINECO and FEDER (MAT2015-69547-R) and the Generalitat de Catalunya (2014SGR188).

\section{Supporting Information}

Supporting Information is attached in a separated file.

\section{References}

(1) Z. Zhang, F. Zhang, X. Jiang, Y. Liu, Z. Guo, J. Leng, Fiber. Polym., 15, 2290 (2014).

(2) R. Scaffaro, F. Lopresti, A. Maio, L. Botta, S. Rigogliuso, G. Ghersi, Compos. Part A-Appl. S., 92, 97 (2017).

(3) R. Scaffaro, A. Maio, F. Lopresti, L. Botta, Polymers, 9, 76 (2017).

(4) M. Leno, R. Su, J. Clarke, Y. Lou, E. Mele, Eur. Polym. J., 87, 241 (2017).

(5) J. Zhu, S. Wei, D. Rutman, N. Haldolaarachchige, D.P. Young, Z. Guo, Polymer, 52, 2947 (2011). 
(6) X. Hu, S. Liu, G. Zhou, Y. Huang, Z. Xie, X. Jing, J. Control. Release, 185, 12 (2014).

(7) Y-E. Miao, S. He, Y. Zhong, Z. Yang, W.W. Tjiu, T. Liu, Electrochim. Acta, 99, 117 (2013).

(8) M. Gorji, A. Jeddi, A.A. Gharehaghaji, J. Appl. Polym. Sci., 125, 4135 (2012).

(9) S. Zhang, W.S. Shim, J. Kim, Mater. Design, 30, 3659 (2009).

(10) P. Tahira, S.A. Arvidson, C.D. Saquing, S.S. Shah, S.A. Khan, Langmuir, 28, 5834 (2012).

(11) R.A. Caruso, J.H. Schattka, A, Greiner, Adv. Mater., 13, 1577 (2001).

(12) J. Yoon, Y-S. Jung, J-M. Kim, Adv. Funct. Mater., 19, 209 (2009).

(13) Y. Zhang, H. Ouyang, C.T. Lim, S. Ramakrishna, Z-M Huang, J. Biomed. Mater. Res. B, 72, 156 (2005).

(14) E. Llorens, L.J. del Valle, R. Ferrán, A. Rodríguez-Galán, J. Puiggalí, J. Polym. Res., 21, 360 (2014).

(15) E. Llorens, S. Bellmunt, L.J. del Valle, J. Puiggalí, J. Polym. Res., 21, 603 (2014).

(16) R. Scaffaro, F. Lopresti, L. Botta, Eur. Polym. J., 96, 266 (2017).

(17) M-X. Ma, Q. Liu, C. Ye, B. Grottkau, B. Guo, Y-F. Song, BioMed Res. Int., Article ID 9251806 (2017).

(18) S. Danti, C. Mota, D. D’alessandro, L. Trombi, C. Ricci, S.L. Redmond, A. De Vito, R. Pini, R.J. Dilley, L. Moroni, S. Berrettini, Hearing Balance Commun., 13, 133 (2015).

(19) B. Ding, E. Kimura, T. Sato, S. Fujita, S. Shiratori, Polymer, 45, 1895 (2004).

(20) G. Mingbo, K. Wang, W. Li, C. Qin, J-J. Wang, L. Dai, Fiber. Polym., 12, 65 (2011).

(21) E. Llorens, H. Ibañez, L.J. del Valle, J. Puiggalí, Mat. Sci. Eng. C, 49, 472 (2015). 
(22) Z. Sun, E. Zussman, A.L. Yarin, J.H. Wendorff, A. Greiner, Adv. Mat., 15, 1929 (2003).

(23) Y. Zhang, Z-M. Huang, X. Xu, C.T. Lim, S. Ramakrishna, Chem. Mater., 16, 3406 (2004).

(24) X-F. Wu, A. Rahman, Z. Zhou, D.D. Pelot, S. Sinha-Ray, B. Chen, S. Payne, A.L. Yarin, J. Appl. Polym. Sci., 129, 1383 (2013).

(25) Z. Sun, E. Zussman, A.L. Yarin, J.H. Wendorff, A. Greiner, Adv. Mater., 15, 1929 (2003).

(26) N. Heidarzadeh, M. Rafizadeh, F.A. Taromi, L.J. del Valle, L. Franco, J. Puiggalí, J. Polymers, 8, 253 (2016).

(27) N. Heidarzadeh, M. Rafizadeh, F.A. Taromi, L.J. del Valle, L. Franco, J. Puiggalí, Polym. Degrad. Stability, 135, 18 (2017).

(28) S. Guo, Q. Ke, H. Wang, X. Jin, Y. Li, J. Appl.Polym. Sci., 128, 3652 (2013).

(29) O. Saligheh, M. Forouharshad, R. Arasteh, R. Eslami-Farsani, R. Khajavi, B. Yadollah Roudbari. J. Polym. Res., 20, 65 (2013).

(30) J. Malda, T.B.F. Woodfield, F. van der Vloodt, C. Wilson, D.E. Martens, J. Tramper, C.A. van Blitterswijk, J. Riesle, Biomaterials, 26, 63 (2005).

(31) L. Moroni, J.R. de Wijn, C.A. van Blitterswijk, J. Biomed. Mater. Res. A, 75, 957 (2005).

(32) H. Qingpu, D.W. Grijpma, J. Feijen, Biomaterials, 24, 1937 (2003).

(33) C.J. Ellison, A. Phatak, D.W. Giles, C.W. Macosko, F.S. Bates, Polymer, 48, 3306 (2007).

(34) S. Chen, W. Yu, J.E. Spruiell, J. Appl. Polym. Sci., 34, 1477 (1987).

(35) S. Kadhiravan, Y. Yichen Fang, D.Y. Chou, S. Sarah Sparks, J. Hibbert, C.J. Ellison, ACS Macro Lett., 1, 960 (2012). 
(36) E.S. Cozza, Q. Ma, O. Monticelli, P. Cebe, Eur. Polym. J., 49, 33 (2013).

(37) L.H. Catalani, G. Collins, M. Jaffe, Macromolecules, 40, 1693 (2007).

(38) T.B.F. Woodfield, J. Malda, J. de Wijn, F. Peters, J. Riesle, C.A. van Blitterswijk, Biomaterials, 25, 4149 (2004).

(39) Y. Ikada, H. Tsuji, Macromol. Rapid Comm., 21, 117 (2000).

(40) S.J. Hollister, Nat. Mater., 4, 518 (2005).

(41) C.T. Laurencin, A.M.A. Ambrosio, M.D. Borden, J.A. Cooper, Annu. Rev. Biomed. Eng., 1, 19 (1999).

(42) E.J. Kim, G.D. Yeo, C.M. Pai, I.K. Kang, J. Biomed. Mater. Res. B, 90, 849 (2009).

(43) V. Tserki, J. Philippou, C. Panayiotou, P. I. Mech. Eng. N., 220, 71 (2006).

(44) E.H. Jeong, S.S. Im, J.H. Youk, Polymer, 46, 9538 (2005).

(45) F. Huang, Q. Wei, J. Wang, Y. Cai, Y. Huang, e-Polymers, 8, 1, 2008.

(46) L.J. del Valle, R. Camps, A. Díaz, L. Franco, A. Rodríguez-Galán, J. Puiggalí, J. Polym. Res., 18, 1903 (2011).

(47) S.L. Shenoy, W.D. Bates, H.L. Frisch, G.E. Wnek, Polymer, 46, 3372 (2005).

(48) R.M. Lum, J. Polym. Sci. A1, 17, 203 (1979).

(49) F. Samperi, C. Puglisi, R. Alicata, G. Montaudo, Polym. Degrad. Stabil., 83,11 (2004).

(50) T. Koshiduka, T. Ohkawa, K. Takeda, Polym. Degrad. Stabil., 79, 1 (2003). 


\section{FIGURE CAPTIONS}

Figure 1. Scheme showing repeat units of involved polymers and the co-electrospinning process.

Figure 2. SEM images at low and high magnification (left and right columns, respectively) of: a) PBT, b) PBSeT, c) PBT/PBSeT and d) co(PBT-PBSeT) electrospun scaffolds.

Figure 3. Frequency distributions of the fiber diameter and Gaussian functions for PBT (a), PBSeT (b), PBT/PBSeT (c) and co(PBT-PBSeT) (d) electrospun scaffolds.

Figure 4. ${ }^{1} \mathrm{H}-\mathrm{NMR}$ spectra of the diferent studied electrospun scaffolds (a) and magnification of the sequence sensitive signals corresponding to the $-\mathrm{OCH}_{2}$ protons of the butanediol unit (b).

Figure 5. DSC heating traces of the different electrospun matrices before (a) and after being slowly cooled $\left(10^{\circ} \mathrm{C} / \mathrm{min}\right)$ from the melt sate (b).

Figure 6. Contact angles (water as a contact liquid) determined for the indicated electrospun scaffolds. ${ }^{*} p<0.05$ vs PBT sample.

Figure 7. Degradation of electrospun PBSeT, PBT, PBT/PBSeT and co-(PBT-PBseT) scaffolds. Plots showing the remaining weight of the different electrospun samples after exposure to universal buffer at $\mathrm{pH} 10$ and $37^{\circ} \mathrm{C}$ (a), universal buffer at $\mathrm{pH} 7$ and $70{ }^{\circ} \mathrm{C}$ (b) and enzymatic medium (pH 7.4 and $37^{\circ} \mathrm{C}$ ) containing lipase from Pseudomonas cepacia (c). SEM images obtained at 30 days of degradation under the conditions indicated in (a), (b) and (c) for fiber matrices of PBT (d), PBSeT (e, f and i), PBT/PBSeT blends (g and j), and co(PBT-PBSeT) (h and k). 
Figure 8. Cell adhesion (a) and proliferation (b) of MDCK epithelial and NRK fibroblast cell lines. ${ }^{*} p<0.05$ vs control.

Figure 9. Cell morphology of MDCK epithelial and NRK fibroblast cell lines grown onto electrospun of PBT, PBSeT, PBT/PBSeT and co(PBT-PBSeT). The asterisk points out the monolayer growth of the cells. The inset shows the spreading of the cells onto the fibers. 


\section{TABLE CAPTIONS}

Table 1. Selected electrospinning parameters for the different studied samples.

Table 2. Main calorimetric data of the first and second heating scans performed with the different studied scaffolds.

Table 3. Thermal degradation data of the different electrospun scaffolds. 
Table 1. Selected electrospinning parameters for the different studied samples.

\begin{tabular}{lcccc}
\hline Sample & $\begin{array}{c}\text { Concentration } \\
(\mathbf{\%}-\mathbf{w} / \mathbf{v})\end{array}$ & $\begin{array}{c}\text { Flow rate } \\
(\mathbf{m L / h})\end{array}$ & $\begin{array}{c}\text { Voltage } \\
\mathbf{( k V )}\end{array}$ & $\begin{array}{c}\text { Distance } \\
(\mathbf{c m})\end{array}$ \\
\hline PBT & 20 & 4 & 20 & 15 \\
PBSeT & 60 & 3 & 15 & 25 \\
PBT/PBSeT & $10 / 10$ & 1.5 & 20 & 13.5 \\
co(PBT-PBSeT) & 20,60 & 4,3 & 20,15 & 7 \\
\hline
\end{tabular}


Table 2. Main calorimetric data deduced from the first and second heating scans performed with the different studied scaffolds.

\begin{tabular}{lcccc}
\hline \multirow{2}{*}{ Sample } & \multicolumn{2}{c}{$\mathbf{1}^{\text {st }}$ Heating Run } & \multicolumn{2}{c}{$\mathbf{2}^{\text {nd }}$ Heating Run } \\
\cline { 2 - 5 } & $\begin{array}{c}\boldsymbol{T}_{\boldsymbol{m}} \\
\left({ }^{\mathbf{O}} \mathbf{C}\right)\end{array}$ & $\begin{array}{c}\Delta \boldsymbol{H}_{\boldsymbol{f}} \\
(\mathbf{J} / \mathbf{g})\end{array}$ & $\begin{array}{c}\boldsymbol{T}_{\boldsymbol{m}} \\
\left({ }^{\circ} \mathbf{C}\right)\end{array}$ & $\begin{array}{c}\Delta \boldsymbol{H}_{\boldsymbol{f}} \\
(\mathbf{J} / \mathbf{g})\end{array}$ \\
\hline PBT & 223 & 55 & 216,219 & 65 \\
PBSeT & 49 & 53 & 46 & 56 \\
PBT/PBSeT & 47,226 & 26,29 & $45,216,223$ & 17,29 \\
co(PBT-PBSeT) & 40,204 & 30,18 & 45,138 & 24,25 \\
\hline
\end{tabular}


Table 3. Thermal degradation data of the different electrospun scaffolds.

\begin{tabular}{lccc}
\hline Sample & $\begin{array}{c}\boldsymbol{T}_{\text {1st step }} \\
\left({ }^{\circ} \mathbf{C}\right)\end{array}$ & $\begin{array}{c}\boldsymbol{T}_{\text {2nd step }} \\
\left({ }^{\circ} \mathbf{C}\right)\end{array}$ & $\begin{array}{c}\text { Char yield } \\
(\mathbf{\%})\end{array}$ \\
\hline PBT & 399.8 & - & 5.1 \\
PBSeT & 407.6 & 455.8 & 2.4 \\
PBT/PBSeT & 403.9 & - & 3.9 \\
co(PBT-PBSeT) & 403.2 & - & 6.5 \\
\hline
\end{tabular}




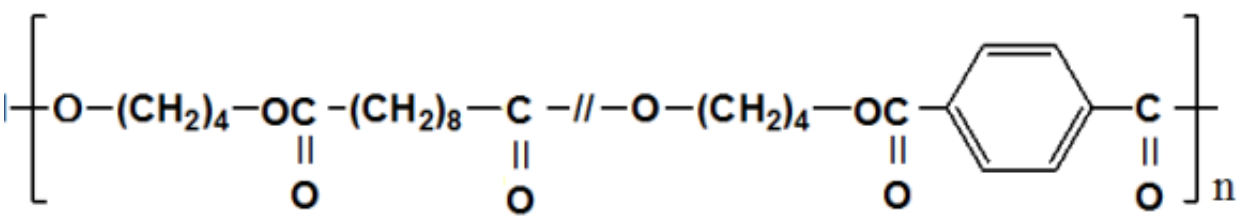

PBSeT
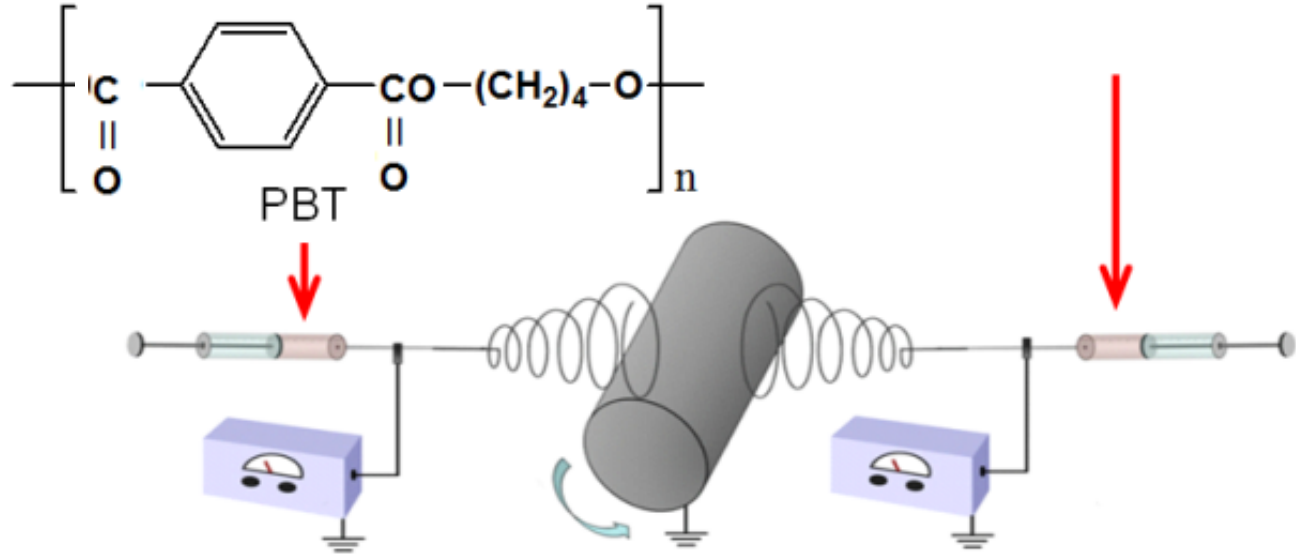

Figure 1. Scheme showing repeat units of involved polymers and the co-electrospinning process. 


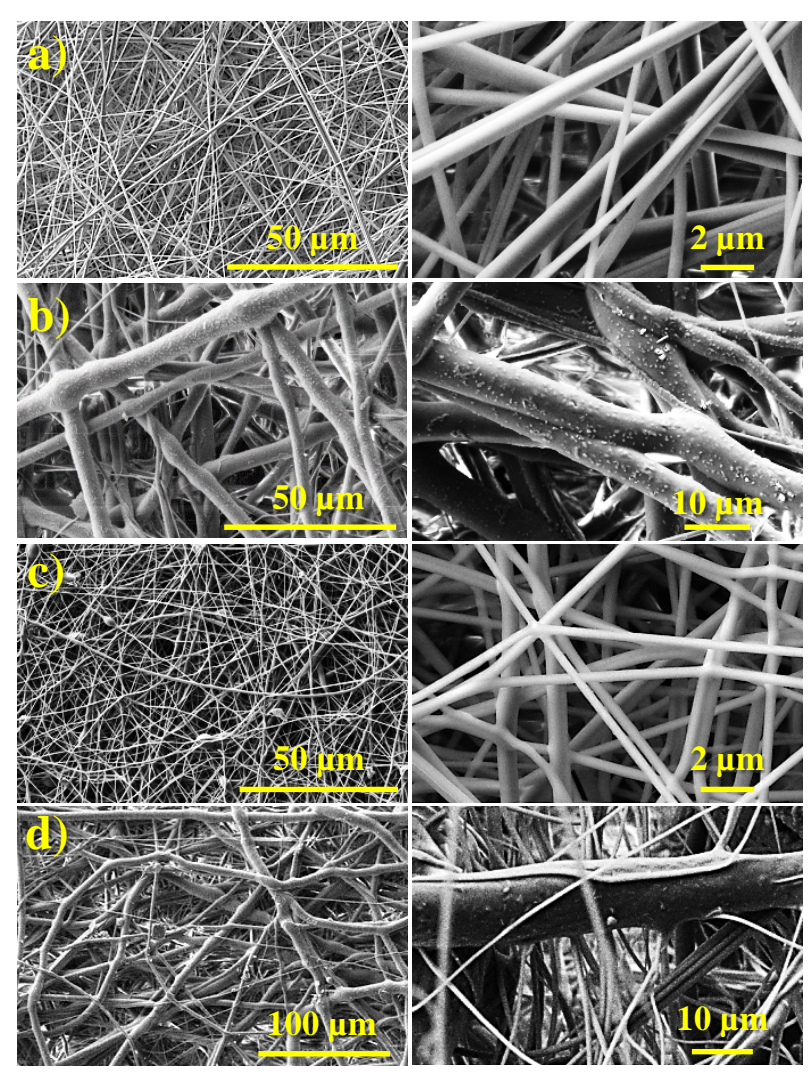

Figure 2. SEM images at low and high magnification (left and right columns, respectively) of: a) PBT, b) PBSeT, c) PBT/PBSeT and d) co(PBT-PBSeT) electrospun scaffolds. 

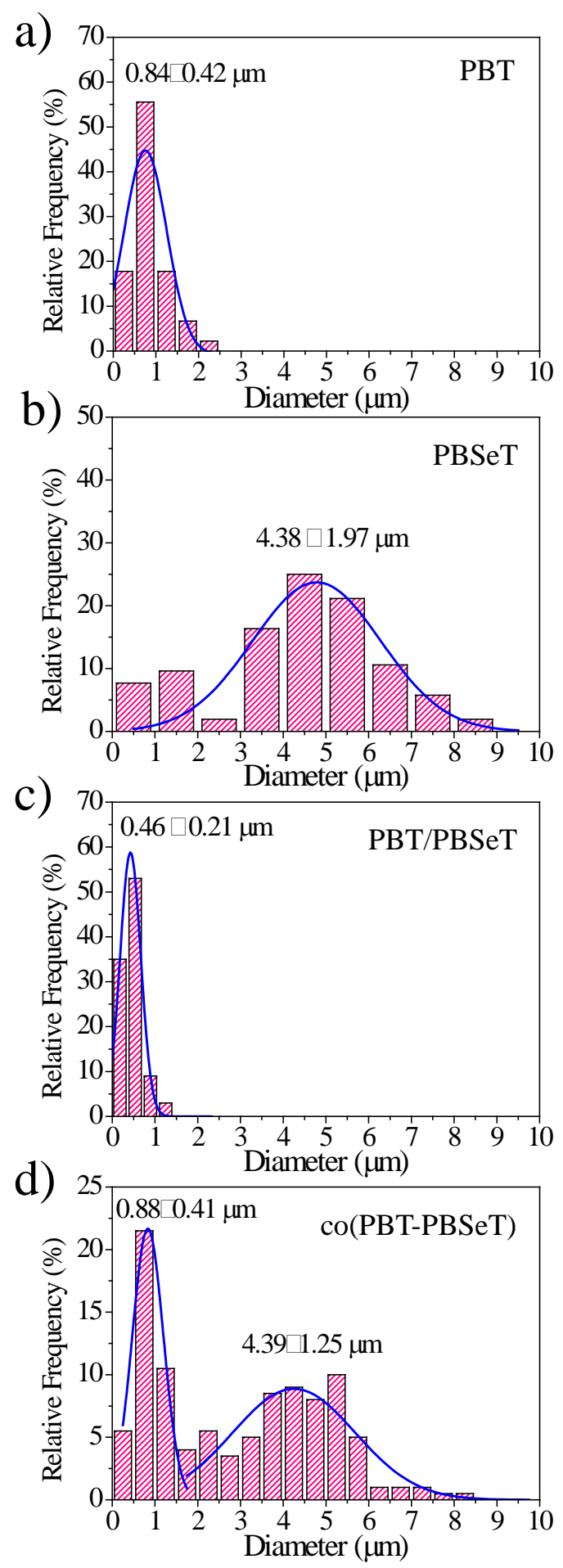

Figure 3. Frequency distributions of the fiber diameter and Gaussian functions for PBT (a), PBSeT (b), PBT/PBSeT (c) and co(PBT-PBSeT) (d) electrospun scaffolds. 

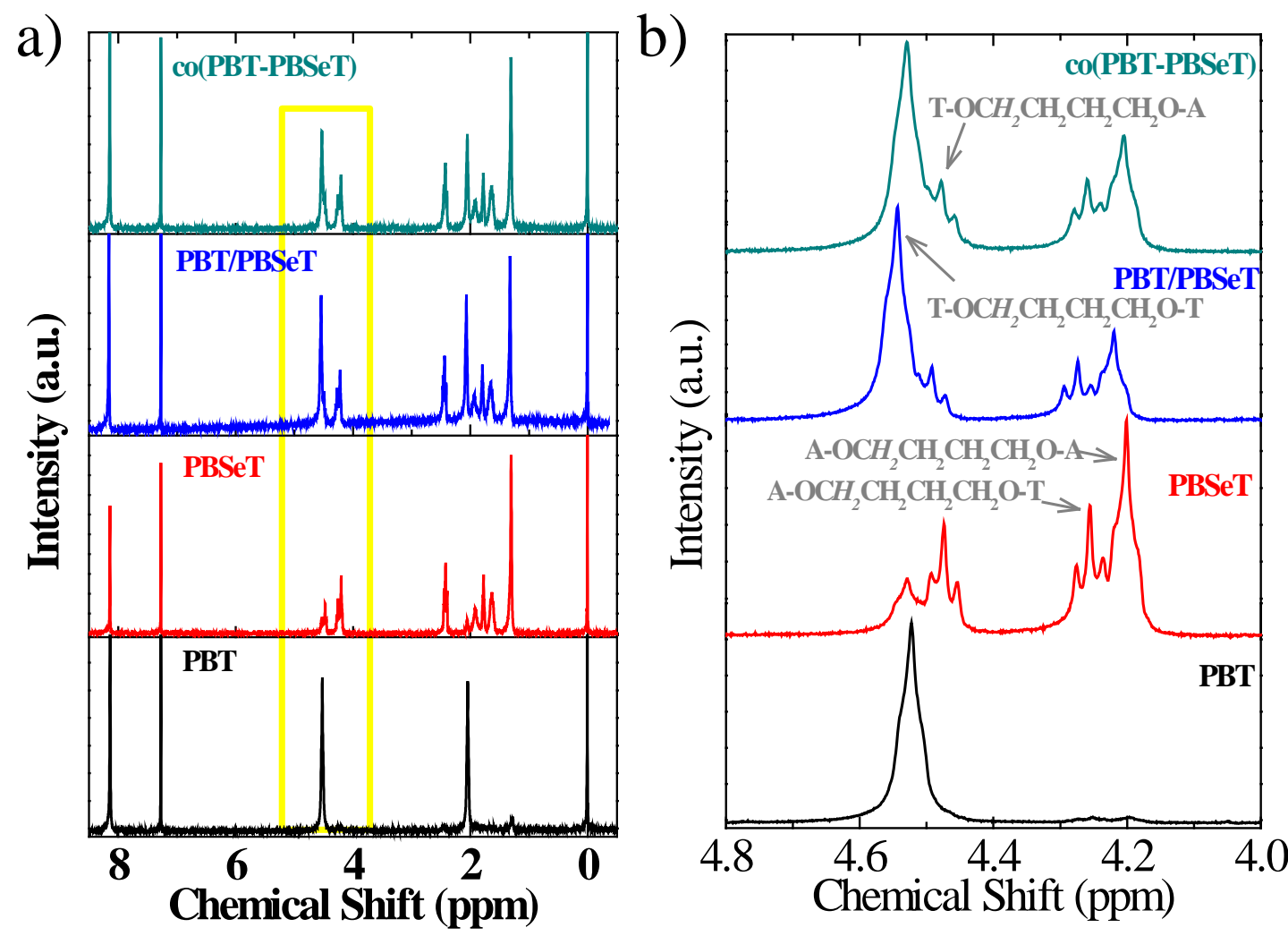

Figure 4. ${ }^{1} \mathrm{H}-\mathrm{NMR}$ spectra of the diferent studied electrospun scaffolds (a) and magnification of the sequence sensitive signals corresponding to the $-\mathrm{OCH}_{2}$ protons of the butanediol unit (b). 

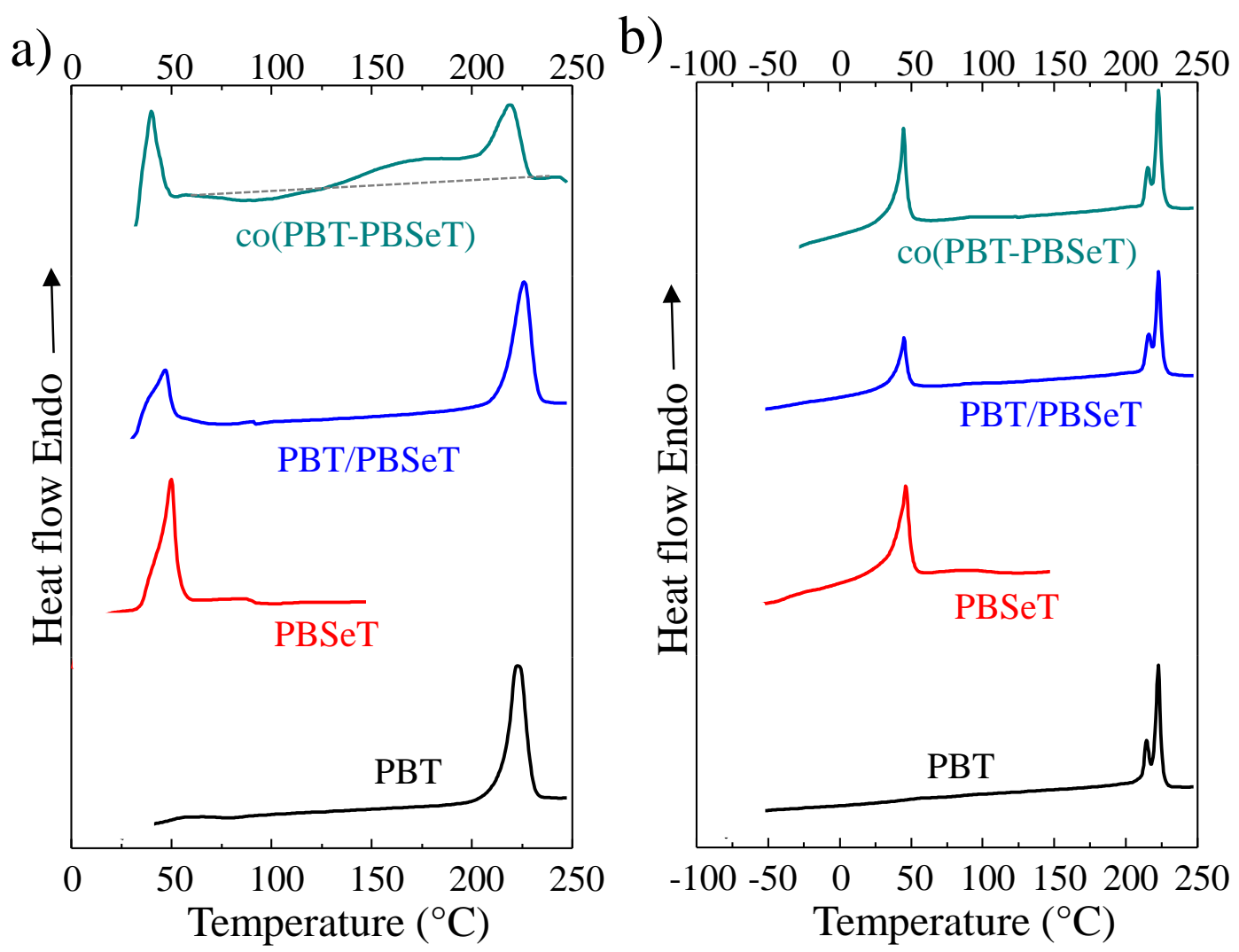

Figure 5. DSC heating traces of the different electrospun matrices before (a) and after being slowly cooled $\left(10^{\circ} \mathrm{C} / \mathrm{min}\right)$ from the melt sate (b). 


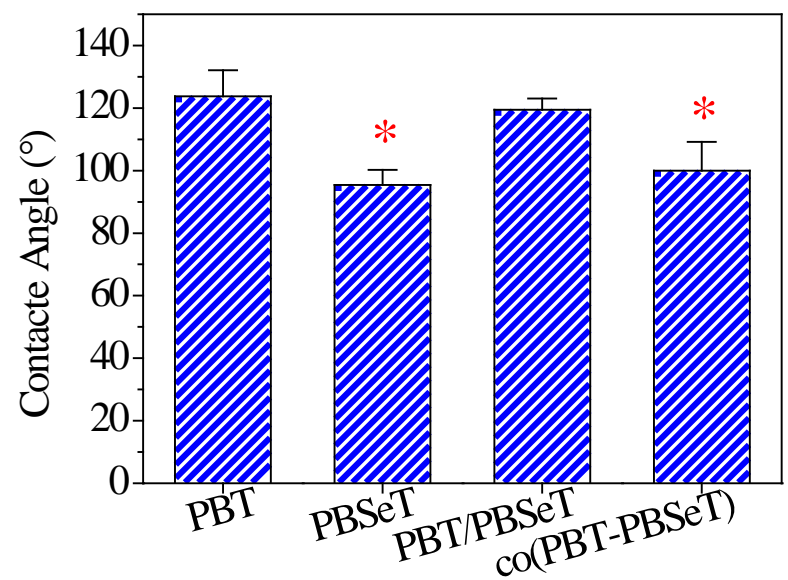

Figure 6. Contact angles (water as a contact liquid) determined for the indicated electrospun scaffolds. ${ }^{*} p<0.05$ vs PBT sample. 

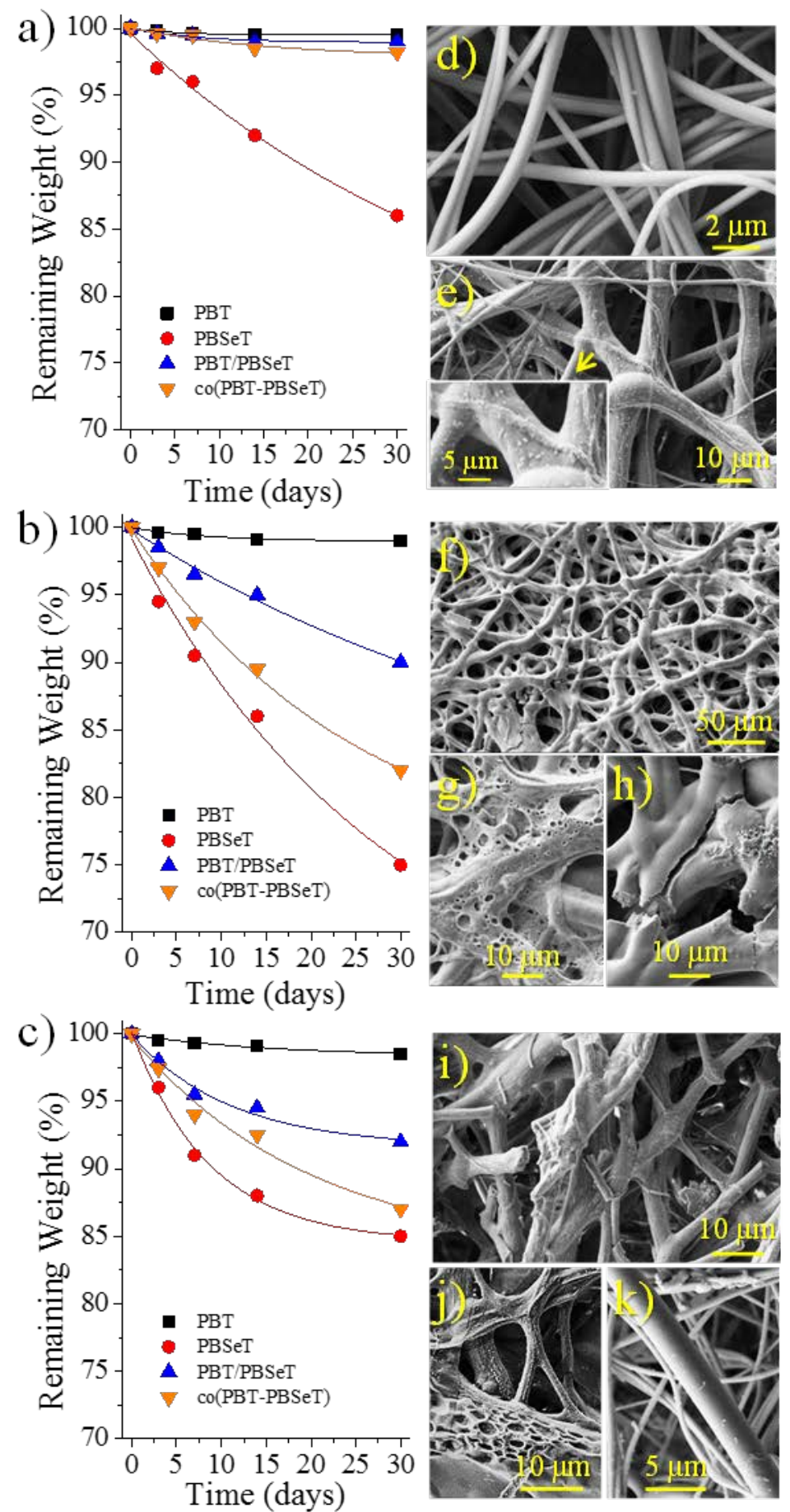

Figure 7. Degradation of electrospun PBSeT, PBT, PBT/PBSeT and co-(PBT-PBseT) scaffolds. Plots showing the remaining weight of the different electrospun samples after exposure to universal buffer at $\mathrm{pH} 10$ and $37^{\circ} \mathrm{C}$ (a), universal buffer at $\mathrm{pH} 7$ and $70{ }^{\circ} \mathrm{C}$ (b) and enzymatic medium ( $\mathrm{pH} 7.4$ and $37^{\circ} \mathrm{C}$ ) containing lipase from Pseudomonas cepacia (c). SEM images obtained at 30 days of degradation under the conditions indicated in (a), (b) and (c) for fiber matrices of PBT (d), PBSeT (e, f and i), PBT/PBSeT blends (g and j), and co(PBT-PBSeT) (h and k). 

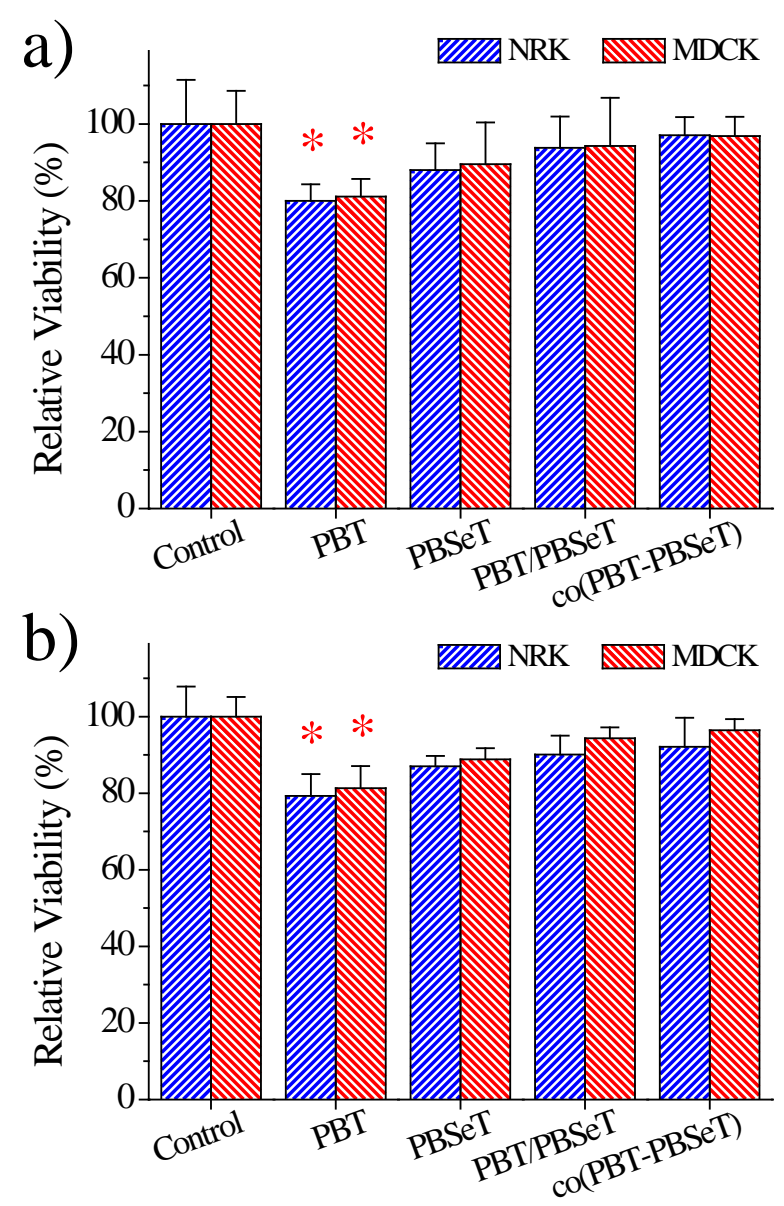

Figure 8. Cell adhesion (a) and proliferation (b) of MDCK epithelial and NRK fibroblast cell lines. ${ }^{*} p<0.05$ vs control. 


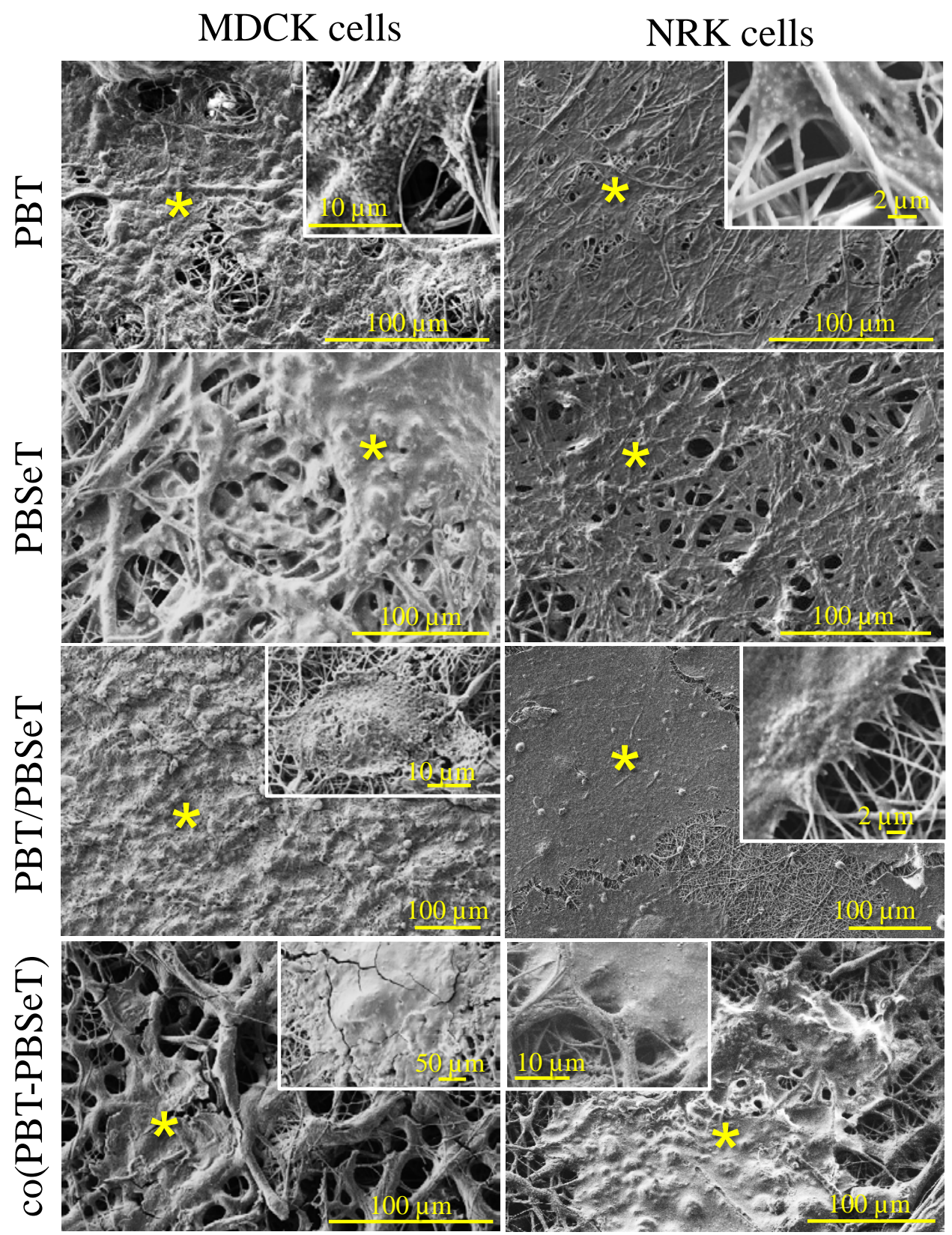

Figure 9. Cell morphology of MDCK epithelial and NRK fibroblast cell lines grown onto electrospun of PBT, PBSeT, PBT/PBSeT and co(PBT-PBSeT). The asterisk points out the monolayer growth of the cells. The inset shows the spreading of the cells onto the fibers. 


\section{Supporting Information}

Improvement of Biodegradability and Biocompatibility of Electrospun Scaffolds of Poly(Butylene Terephthalate) by Incorporation of Sebacate Units

Nina Heidarzadeh ${ }^{1}$, Luis J. del Valle ${ }^{2,3}$, Lourdes Franco ${ }^{2,3}$, Jordi Puiggali $\mathbf{1}^{2,3 *}$

\footnotetext{
${ }^{1}$ Department of Polymer Engineering and Color Technology, Amirkabir University of Technology, PO Box 15875-441, Tehran, IRAN

${ }^{2}$ Chemical Engineering Department, Escola d'Enginyeria de Barcelona Est-EEBE, Av. Eduard Maristany 10-14, Barcelona 08019, SPAIN

${ }^{3}$ Barcelona Research Center for Multiscale Science and Engineering. Universitat Politècnica de Catalunya, Escola d'Enginyeria de Barcelona Est-EEBE, Av. Eduard Maristany 10-14, Barcelona 08019, SPAIN
} 


\section{Synthesis of the PBSeT copolyester}

A 30:70 molar ratio mixture of TA and SeA was introduced into a stainless reactor, together with an excess of 1,4-butanediol (i.e. a sebacic:butanediol molar ratio of 1.17:1). The temperature was then set to $200{ }^{\circ} \mathrm{C}$ under a nitrogen atmosphere. Water byproduct was separated during the esterification step and removed in a condenser. The reaction was stopped when no more water was recovered.

In the second polycondensation step, TBT catalyst was added (1.4 mmol for $1 \mathrm{~mol}$ of dicarboxylic acid). The reaction mixture was initially kept for $10 \mathrm{~min}$ at $200{ }^{\circ} \mathrm{C}$ under a nitrogen atmosphere and then vacuum (20 mbar) was slowly applied over a time period of about 20 min to minimize oligomer sublimation, which is a potential problem during melt polycondensation. The temperature was finally increased to $250-255{ }^{\circ} \mathrm{C}$ for an additional time period of 2.5 hours. The copolymer was purified through precipitation with 1,1,1,3,3,3-hexafluoroisopropanol (HFIP) solution, being subsequently washed with water, methanol and ether, several times. Molecular weight was determined by gel permeation chromatography (GPC).

A Shimadzu LC-8A chromatograph (Tokyo, Japan) equipped with the LC solution V.1.25 SP2 software from Shimadzu was employed together with a PL HFIP gel column (Polymer Lab, Germany) and a refractive index detector (Shimadzu RID-10A). Polymethyl methacrylate standards were used to estimate number and weight average molecular weights of 8,600 and 19,300 g/mol, respectively. ${ }^{1} \mathrm{H}$ NMR spectra allowed determining a 69 mol-\% (i.e. 75 wt-\%) of butylene sebacate units as explained in the main document. 


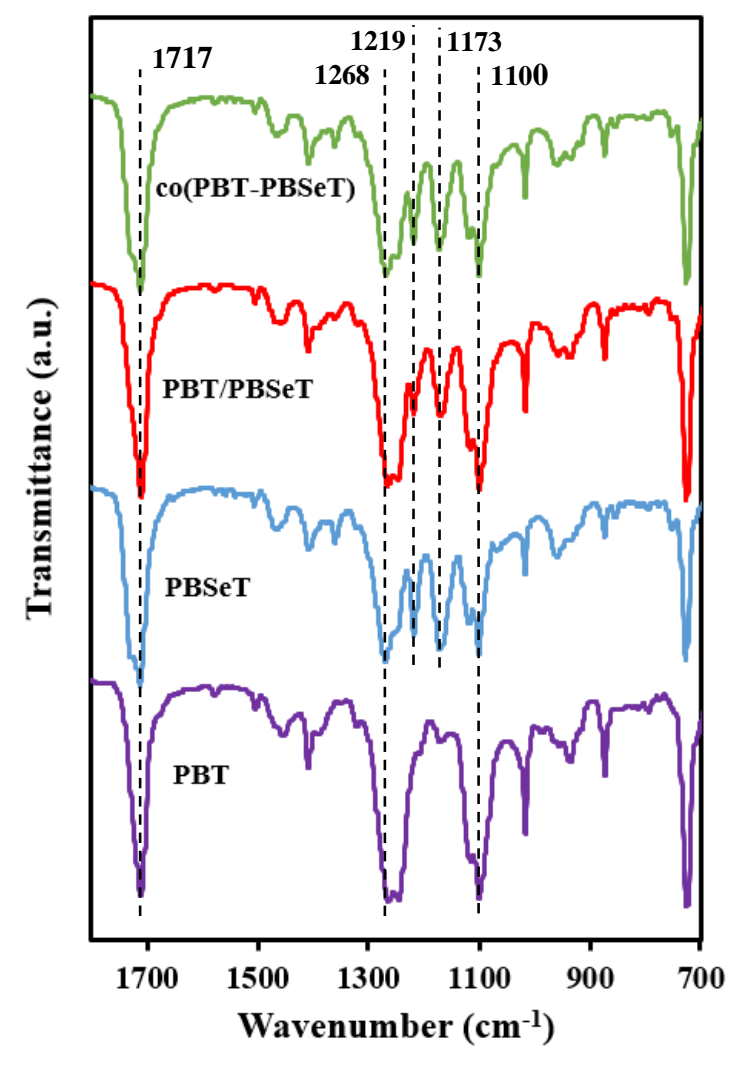

Figure S1. FTIR of the indicated electrospun scaffolds. All spectra have been normalized considering their common and strongest $\mathrm{C}=\mathrm{O}$ band at $1717 \mathrm{~cm}^{-1}$. 


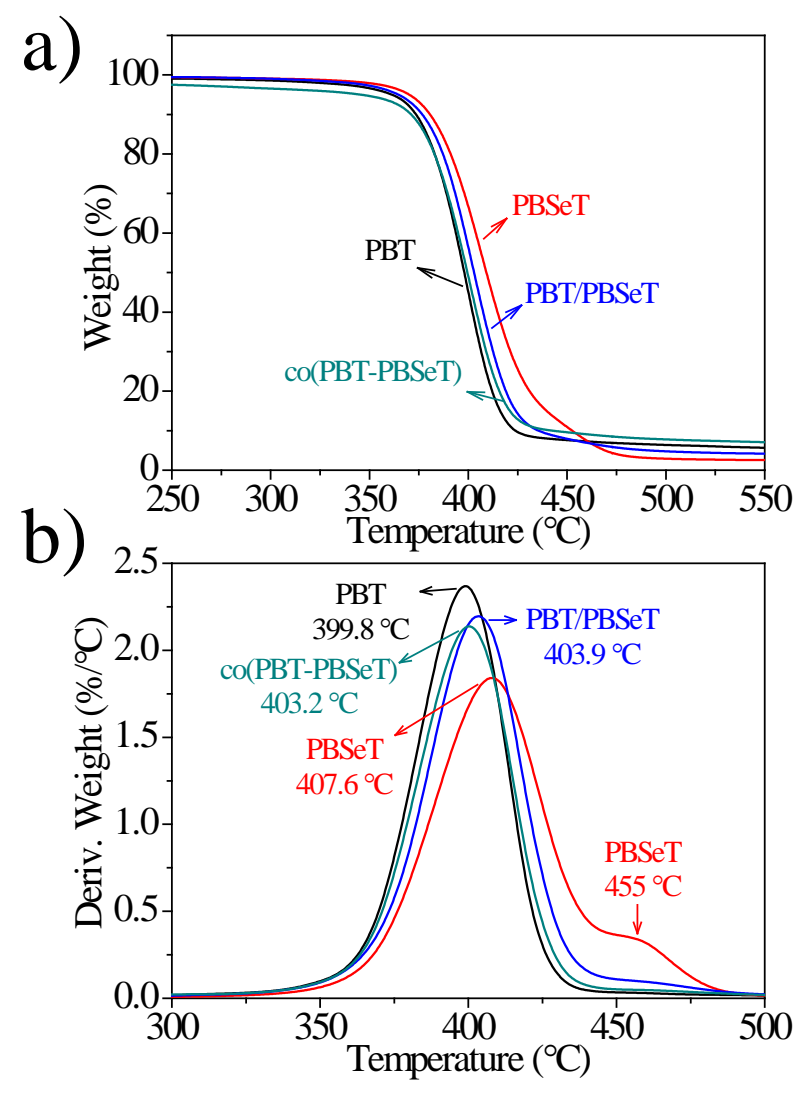

Figure S2. TGA and DTGA thermogravimetric curves for PBT, PBSeT, PBT/PBSeT and co(PBT-PBSeT) electrospun scaffolds. 


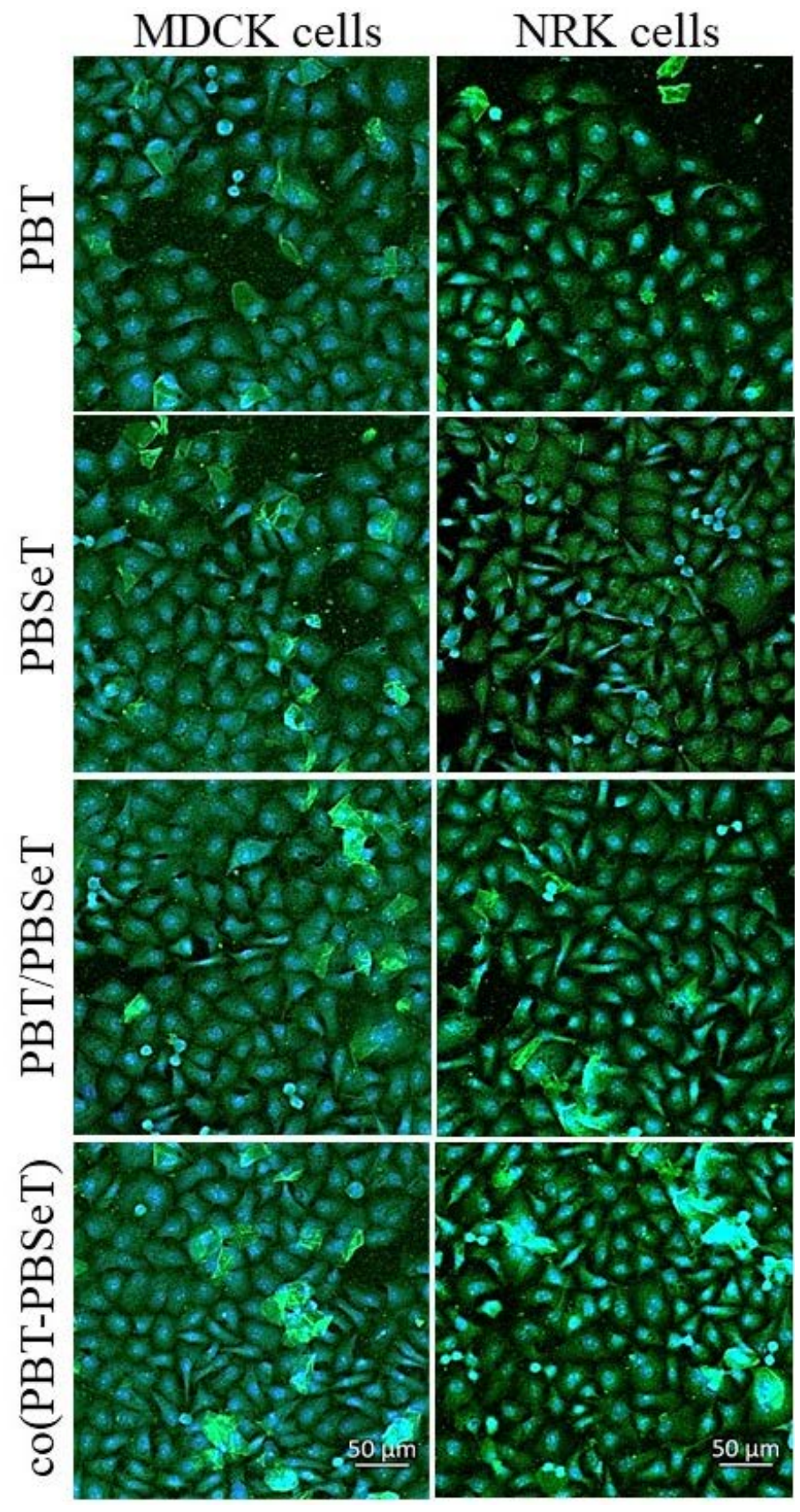

Figure S3. MDCK epithelial and NRK fibroblast cells grown onto electrospun matrices of PBT, PBSeT, PBT/PBSeT and co(PBT-PBSeT). The actin filaments are observed in green and nuclei in blue color. 